\title{
Subpicosecond Magnetization Reversal across Ferrimagnetic Compensation Points
}

\author{
C. D. Stanciu, ${ }^{1}$ A. Tsukamoto, ${ }^{2}$ A. V. Kimel, ${ }^{1}$ F. Hansteen, ${ }^{1}$ A. Kirilyuk, ${ }^{1}$ A. Itoh, ${ }^{2}$ and Th. Rasing ${ }^{1}$ \\ ${ }^{1}$ Institute for Molecules and Materials, Radboud University Nijmegen, Toernooiveld 1, 6525 ED Nijmegen, The Netherlands \\ ${ }^{2}$ College of Science and Technology, Nihon University, 7-24-1 Funabashi, Chiba, Japan \\ (Received 15 May 2007; revised manuscript received 14 August 2007; published 19 November 2007)
}

\begin{abstract}
Subpicosecond magnetization reversal is experimentally demonstrated by ultrafast heating of a ferrimagnet across its compensation points, under an applied magnetic field. While the reversal is initiated by crossing the magnetization compensation temperature, the short reversal time is related to the angular momentum compensation, where the dynamics of the system is highly accelerated owing to the divergence of the gyromagnetic ratio. These results demonstrate the feasibility of subpicosecond magnetization reversal previously believed impossible.
\end{abstract}

DOI: 10.1103/PhysRevLett.99.217204

The fundamental limit of the magnetization reversal time is presently one of the most intriguing subjects in the physics of magnetism, with crucial consequences for magnetic recording and information processing [1-13]. Traditionally, to reverse the magnetization, a magnetic field is applied in the opposite direction of the magnetization. In that case, the magnetization reversal is achieved by domain-wall motion. Alternatively, by applying a magnetic field perpendicular to the magnetization, a coherent "precessional switching" occurs, known as the fastest way to reverse magnetization [2-5]. A fascinating and even faster way of switching has recently been demonstrated in micromagnetic simulations via ultrafast switching of vortex cores [6]. However, it was predicted that there is a natural limit of magnetization switching on the picosecond time scale, beyond which magnetization reversal becomes nondeterministic [7]. Thus, finding new approaches to reverse magnetization in a reproducible way on a time scale shorter than picoseconds is a fundamental challenge with important consequences for technology.

Ferrimagnetism represents one of the keys to access the field of subpicosecond magnetization reversal. This is because a ferrimagnet may possess both magnetization compensation temperature $T_{\mathbf{M}}$ and angular momentum compensation temperature $T_{\mathrm{A}} \cdot T_{\mathrm{M}}$ is the result of a different temperature dependence of the two sublattice magnetizations and represents the point where the magnetizations of these two antiparallel-coupled sublattices are equal and cancel each other. A small difference between the gyromagnetic ratio of the two sublattices leads to an angular momentum compensation point $T_{\mathbf{A}}$ slightly above $T_{\mathbf{M}}$ [11,14]. At $T_{\mathbf{A}}$, application of a magnetic field should instantaneously flip the magnetization. The reason for this is that at $T_{\mathrm{A}}$, both the frequency of the magnetization precession [11] and the domain-wall velocity $[15,16]$ increase strongly due to the divergence of the gyromagnetic ratio [17]. In other words, at $T_{\mathrm{A}}$, magnetization can be regarded as a mechanical system with no inertia which can be moved by the slightest torque. However, to verify this ultrafast switching, instantaneous application of a magnetic field is required which in real experiments is not
PACS numbers: 75.40.Gb, 75.50.Gg, 75.60.Jk, 76.50.+g

feasible. Instead, a dc magnetic field can be applied to the ferrimagnet parallel to the original magnetization direction, at a temperature $T<T_{\mathbf{M}}$. When the temperature of the ferrimagnet increases above both $T_{\mathbf{M}}$ and $T_{\mathbf{A}}$, its magnetization will reverse $[18,19]$. Thus, a femtosecond (fs) laser pulse heating the sample might act as an instantaneously applied magnetic field, allowing the investigation of the magnetization reversal speed at $T_{\mathbf{A}}$. An interesting ferrimagnetic system for this study is the family of amorphous rare-earth (RE)-transition-metal (TM) alloys, such as $\mathrm{GdFeCo}$, for which the compensation points can be tuned in a wide temperature range by simply varying its composition [20]. However, due to the localized character of the spins responsible for magnetism in the $\mathrm{RE}$ ferrimagnetic sublattice, it is not clear whether its magnetization can be changed by laser pulses on a fs time scale [21].

In this Letter, we present experimental evidence that an ultrafast laser-induced heating of the GdFeCo ferrimagnetic system over its compensation points under an applied magnetic field results in a subpicosecond magnetization reversal. Additionally, the observed ultrafast switching implies that the magnetization compensation point is reached on the subpicosecond time scale, thus proving that both itinerant TM and localized RE spins are heated on the fs time scale.

The amorphous alloy GdFeCo is a well suited material for this study. In this ferrimagnetic alloy, the FeCo sublattice is antiferomagnetically coupled to the Gd sublattice in a collinear alignment [22]. The samples used in this study were grown by magnetron sputtering in the following multilayer structure: glass/AlTi $(10 \mathrm{~nm}) / \mathrm{SiN}(5 \mathrm{~nm}) /$ $\mathrm{Gd}_{23} \mathrm{Fe}_{73.64} \mathrm{Co}_{3.36}(20 \mathrm{~nm}) / \mathrm{SiN}(60 \mathrm{~nm})$. The AlTi layer serves as a heat sink. Here, the ferrimagnetic GdFeCo layer is characterized by a strong perpendicular magnetic anisotropy and a Curie temperature of about $525 \mathrm{~K}$. Given the sample composition, the temperature dependence of the hysteresis loop shows a divergence of the coercive field $H_{\mathbf{c}}$ at about $370 \mathrm{~K}$, which indicates the magnetization compensation temperature $T_{\mathbf{M}} \cdot T_{\mathbf{A}}$ occurs at a temperature of about $50 \mathrm{~K}$ above $T_{\mathbf{M}}[11,14]$. 
To initiate and investigate the magnetization reversal in GdFeCo over its compensation points, we have used an alloptical pump-probe technique employing an amplified Ti:Sapphire laser system with 40 fs laser pulses at a central wavelength of $800 \mathrm{~nm}$ and a repetition rate of $1 \mathrm{kHz}$. The magnetization response resulting from the heating by the pump pulse was investigated by a less intense probe beam, which monitored in time the changes in the Faraday rotation. At nearly normal incidence, the linearly polarized beams were focused on the sample to a spot of $200 \mu \mathrm{m}$ diameter for the pump and $40 \mu \mathrm{m}$ for the probe beam. The present geometry is sensitive to the variation in the out-ofplane component of the magnetization, $\mathbf{M}_{z}$. Note that at the $800 \mathrm{~nm}$ probe wavelength, the Faraday signal is mainly given by the FeCo sublattice [11]. The measurements were carried out at room temperature, thus just below $T_{\mathbf{M}}$. During this stroboscopic experiment, to ensure the same initial magnetic state, a magnetic field $\mathbf{H}_{\mathrm{ext}}=0.3 T>$ $H_{c}^{\text {room }}$ was applied at an angle $\theta_{\text {ext }}=30^{\circ}$ from the plane of the sample. Here, $H_{c}^{\text {room }}$ denotes the coercive field at room temperature.

Figure 1 shows that the variation of the time-resolved Faraday signal of GdFeCo has a strong laser fluence dependence. In particular, following laser excitation with a low pump fluence $\left(2.5 \mathrm{~mJ} / \mathrm{cm}^{2}\right)$, a steplike change in the Faraday signal is observed. The steplike process is due to the laser-induced change in the magnitude of both magnetization and magneto-crystalline anisotropy [23]. Subsequently, the magnetization relaxes back towards the initial state. Note that this pump fluence induces an average increase of the local temperature in the sample with $\approx$ $50 \mathrm{~K}$ [11]. As the laser fluence increases, one can observe a gradual increase in the transient Faraday signal. Interestingly, after high pump fluence excitation $\left(6.29 \mathrm{~mJ} / \mathrm{cm}^{2}\right)$, as the heat is diffused out of the sample, the magnetic system relaxes towards the opposite direction first, before cooling down and reversing back to the initial state.

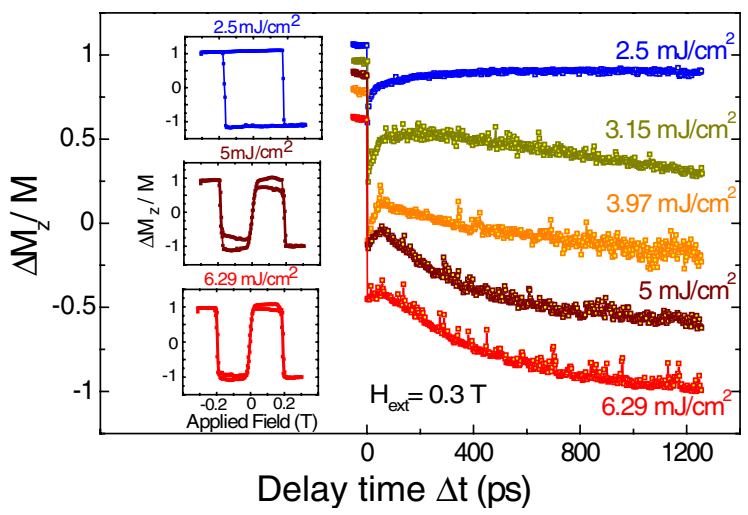

FIG. 1 (color online). Transient Faraday effect traces at different pump fluences. The variation of the magneto-optical signal was plotted relative to the total Faraday rotation at room temperature. The data are offset for clarity. Insets: hysteresis loops measured at negative delay for distinct pump fluences.
To investigate the atypical magnetization dynamics observed at pump fluences higher than $2.5 \mathrm{~mJ} / \mathrm{cm}^{2}$, we have measured hysteresis loops at negative delay for different pump fluences, shown as insets in Fig. 1. Here, a negative delay time implies that the probe pulses were probing the magnetic state long after the pump excitation ( $1 \mathrm{~ms}$ ). While at a $2.5 \mathrm{~mJ} / \mathrm{cm}^{2}$ laser fluence, the measured hysteresis shows no changes due to the laser pumping, the situation changes drastically for the higher pump fluences where anomalous hysteresis loops are observed. To explain these hysteresis loops, we note that at high laser fluences, the sample is heated above $T_{\mathbf{M}}$. As shown in Fig. 2(a), depending on the strength of $\mathbf{H}_{\mathrm{ext}}$, one can distinguish two different scenarios: (A) the applied magnetic field is stronger than the coercive field at the initial temperature $\left(\mathbf{H}_{\mathrm{ext}}>\right.$ $\left.H_{c}^{\mathrm{room}}\right)$; (B) the applied magnetic field is weaker than the coercive field $\left(\mathbf{H}_{\mathrm{ext}}<H_{c}^{\mathrm{room}}\right)$.

(A) When $\mathbf{H}_{\text {ext }}>H_{c}^{\text {room }}$, below $T_{\mathbf{M}}$ the Gd magnetic moment, being larger than that of $\mathrm{FeCo}$, aligns parallel to the external field $\mathbf{H}_{\mathrm{ext}}$. A sudden laser-induced increase of
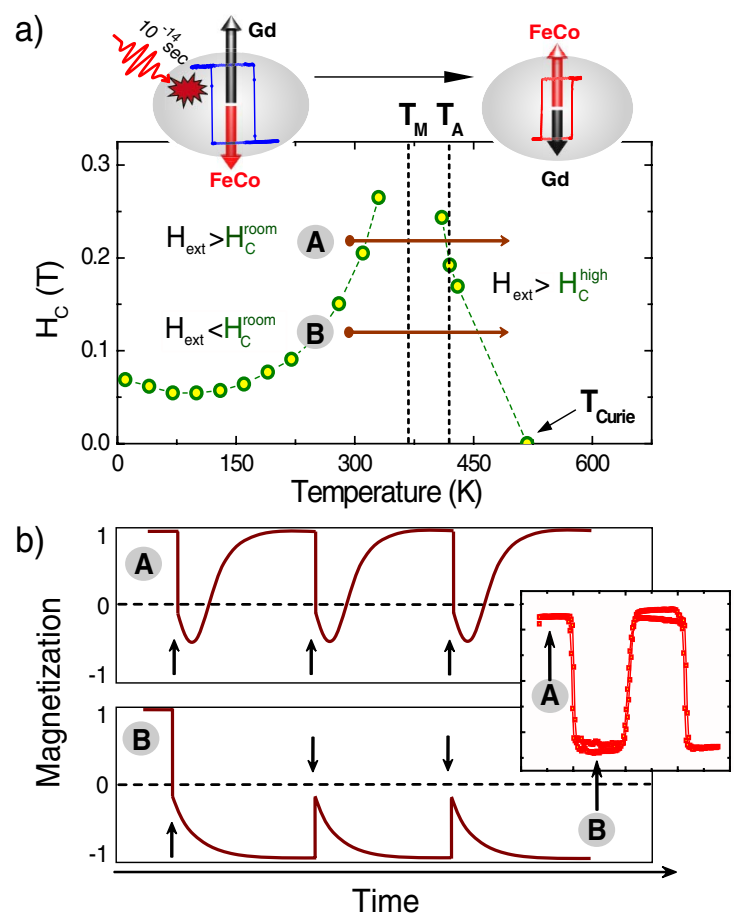

FIG. 2 (color online). (a) Temperature dependence of the coercive field $H_{\mathbf{c}}$ in the GdFeCo sample. The divergence of $H_{\mathbf{c}}$ indicates the presence of $T_{\mathbf{M}}$. The inset of the figure shows schematically the ferrimagnetic system in an applied magnetic field below and above $T_{\mathbf{M}}$. Depending on the strengths of the applied magnetic field, two different dynamic regimes can be distinguished: (A) for $\mathbf{H}_{\mathrm{ext}}>H_{c}^{\mathrm{room}}$ and (B) for $\mathbf{H}_{\mathrm{ext}}<H_{c}^{\mathrm{room}}$. (b) Qualitative description of the magnetization dynamics induced by the first pump pulses, at a $6.29 \mathrm{~mJ} / \mathrm{cm}^{2}$ fluence, for the two different conditions $[(\mathbf{A})$ and $(\mathbf{B})]$. The arrows indicate the time when the pump pulses hit the sample $(1 \mathrm{~ms}$ between two pulses). The inset shows the hysteresis loop measured at negative delay for the $6.29 \mathrm{~mJ} / \mathrm{cm}^{2}$ pump fluence. 
the sample temperature above $T_{\mathbf{M}}$, where the FeCo moment becomes dominant, induces switching of the ferrimagnetic system, as long as the coercive field at high temperature $H_{c}^{\text {high }}<\mathbf{H}_{\text {ext }}$. In our experiments, this occurs at the $6.29 \mathrm{~mJ} / \mathrm{cm}^{2}$ laser fluence that corresponds to an increase of the local temperature of about $190 \mathrm{~K}$. After the pump pulse, and as long as the spin temperature is still above $T_{\mathbf{M}}$, the FeCo sublattice relaxes towards a metastable opposite state. When the sample cools down below $T_{\mathbf{M}}$, because $\mathbf{H}_{\mathrm{ext}}>H_{c}^{\text {room }}$, the recovered magnetization will eventually switch back restoring the initial condition. The same type of dynamics will occur for every pump pulse [see Fig. 2(b) -upper panel]. This clarifies the magnetization dynamics shown in Fig. 1 for a laser fluence of $6.29 \mathrm{~mJ} / \mathrm{cm}^{2}$, where the magnetization is observed to relax towards the opposite magnetic state.

(B) A different scenario is taking place when $H_{c}^{\text {high }}<$

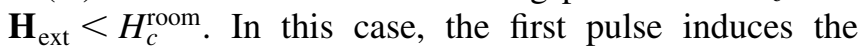
reversal of the magnetization which after the heat is diffused away can not reverse back to the initial state as in the previous case, because $\mathbf{H}_{\text {ext }}<H_{c}^{\text {room }}$. Thus, the magnetization dynamics initiated by the next pulses occur in this opposite magnetic state as shown in the lower panel of Fig. 2(b). In a static view, when the applied magnetic field is weaker than the coercive field at room temperature, the magnetization of the ferrimagnetic system $\mathrm{GdFeCo}$ is reversed oppositely to the applied magnetic field. This explains the anomalous hysteresis loops observed at negative delay for laser fluences such as $6.29 \mathrm{~mJ} / \mathrm{cm}^{2}$ and demonstrates the magnetization reversal over $T_{\mathbf{M}}$ by laserinduced heating.

The hysteresis loop measured at a pump fluence of $5 \mathrm{~mJ} / \mathrm{cm}^{2}$ shows a residual hysteresis in the (B) field range. This happens because at lower pump fluence, part of the probed area does not fulfill the condition $\mathbf{H}_{\mathrm{ext}}>$ $H_{c}^{\text {high }}$, resulting in an inhomogeneous switching. This also explains the observed gradual increase of the transient Faraday effect with increasing pump fluence shown in Fig. 1.

A fundamental question arising from the above demonstrated magnetization switching is: How fast is this switching process? To answer this question, we have measured hysteresis loops at different time delays after the pump pulses heat the sample with a fluence of $6.29 \mathrm{~mJ} / \mathrm{cm}^{2}$. The results are shown in Fig. 3. Since only for the condition $\mathbf{H}_{\text {ext }}>H_{c}^{\text {room }}$ the system switches and returns to the initial state for each pump pulse, one should focus the attention on the (A) field range of the measured hysteresis. One can clearly observe that in this field range, the measured Faraday signal changes its sign after about 700 fs. The sign change reflects the change of the FeCo-sublattice direction towards the applied magnetic field, as the spin temperature of the ferrimagnetic system increases over $T_{\mathbf{M}}$ in the probed area. This observation unambiguously demonstrates that the magnetization reversal takes place on a

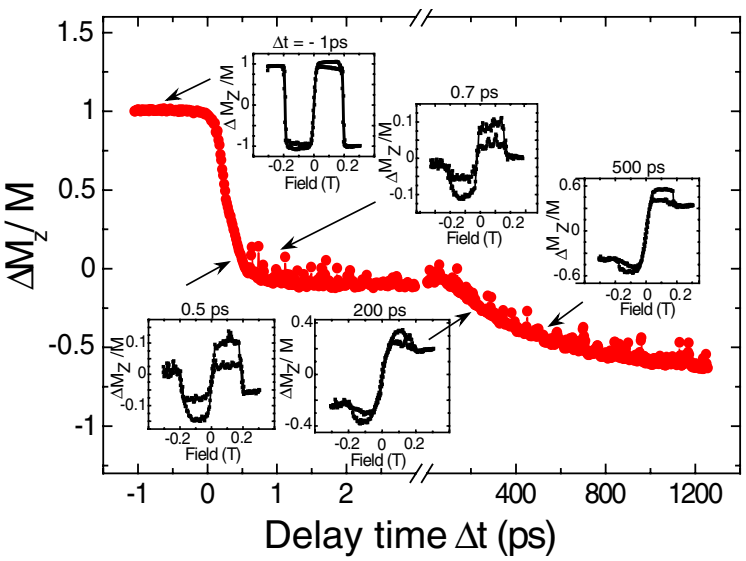

FIG. 3 (color online). Transient magnetization reversal dynamics measured for a pump fluence of $6.29 \mathrm{~mJ} / \mathrm{cm}^{2}$. Insets show hysteresis loops measured at distinct pump-probe delays. The loops demonstrate the magnetization reversal after about $700 \mathrm{fs}$.

subpicosecond time scale. Note that this reversal time is considerably faster than that found in $\mathrm{GdFeCo}$ at temperatures above the compensation points [24]. On the other hand, the growth of the reversed domain to its full $100 \%$ is determined by the cooling rate and takes place at a much longer time scale.

This ultrafast reversal time can be related to the angular momentum compensation $T_{\mathbf{A}}[18,19]$. In $\mathrm{GdFeCo}$, the difference between the gyromagnetic ratios of the $\mathrm{Gd}$ and FeCo sublattices induces a separation in temperature between the magnetization and the angular momentum compensation points of about $50 \mathrm{~K}$, where $T_{\mathbf{A}}>T_{\mathbf{M}}[11,14]$. The shift of $T_{\mathbf{A}}$ with respect to $T_{\mathbf{M}}$ represents an advantage for the present experiment, insuring a $H_{c}<\mathbf{H}_{\mathrm{ext}}$ at $T_{\mathrm{A}}$ [see Fig. 2(a)]. A laser fluence of $6.29 \mathrm{~mJ} / \mathrm{cm}^{2}$ induces a heating of about $190 \mathrm{~K}$, which implies that the laser pulses locally elevate the sample's temperature to above both $T_{\mathbf{M}}$ and $T_{\mathrm{A}}$. Because the effective gyromagnetic ratio of the ferrimagnetic system $\gamma_{\text {eff }}$ is inversely proportional to the total angular momentum of the system $\left(\gamma_{\mathrm{eff}}=\mathbf{M} / \mathbf{A}\right)$, theoretically $\gamma_{\text {eff }}$ diverges when $T_{\mathbf{A}}(\mathbf{A}=0)$ is reached [17]. Both magnetization precession frequency [11] and domain-wall velocity $[15,16]$ are proportional to this effective gyromagnetic ratio. Thus, in the vicinity of $T_{\mathrm{A}}$, a many fold increase of the frequency of the spin precession and domain-wall velocity are expected. Consequently, the magnetization reversal will indeed be highly accelerated at $T_{\mathrm{A}}$, leading to an ultrafast magnetization. On the other hand, at $T_{\mathbf{A}}$, also the effective Gilbert damping of the ferrimagnetic system shows a peak [11]. An increase of this parameter results in a decrease of the domain-wall velocity [16] and thus a slower switching speed. This might represent the mechanism that limits the switching time at $T_{\mathrm{A}}$ to $700 \mathrm{fs}$ as observed in our experiments.

What are the implications of the observed subpicosecond magnetization reversal? Although it is well known that 


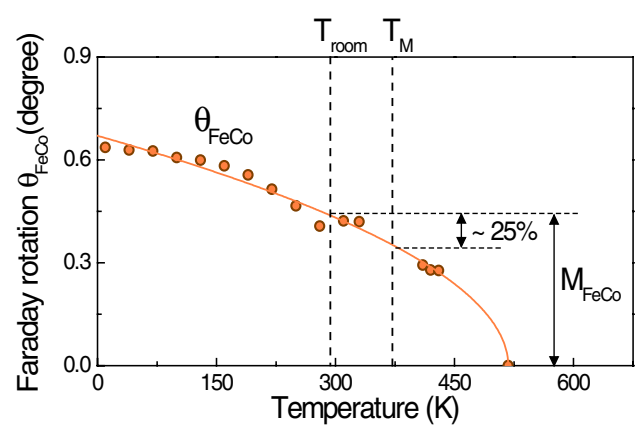

FIG. 4 (color online). Faraday rotation $\theta_{\text {FeCo }}$ at $\lambda=805 \mathrm{~nm}$ as a function of temperature representing the temperature dependence of the FeCo sublattice magnetization. Here, $\mathbf{M}_{\mathbf{F e C o}}$ represents the FeCo sublattice magnetization at the room temperature.

ultrafast laser excitation of itinerant ferromagnets as Co, $\mathrm{Ni}$, or Fe leads to a demagnetization on the femtosecond time scale [1,25], little is known about how fast the magnetic moments in metals as Gd can be excited, with photon energies in the visible range. This is because in $\mathrm{Gd}$, the optically excited electrons of the $5 d 6 s$ band carries only $\approx 9 \%$ of the total moment while the localized $4 f$ electrons dominate the magnetic spin moment. The localization of the magnetic moment together with the weak spin-orbit coupling characteristic for Gd indicates that the transfer of the photon energy to the localized states should be a slow process [26]. In this context, it was recently claimed that laser excitation of a CoGd sample resulted in an independent excitation of the Co sublattice only [27]. In contrast to this claim, we have demonstrated here a subpicosecond magnetization reversal over the compensation points, which implies that both $\mathrm{Gd}$ and FeCo-sublattice magnetizations are considerably reduced on the subpicosecond time scale. In particular, an increase of the spin temperature above $T_{\mathbf{M}}$ in our experiments requires a partial demagnetization of the FeCo sublattice of $\sim 25 \%$ (see Fig. 4). Consequently, the magnetization of the Gd sublattice, which below $T_{\mathbf{M}}$ is larger than that of $\mathrm{FeCo}$, must be reduced by more than $25 \%$. For Gd, this represents a reduction of the magnetic moment far larger than that supplied by the itinerant $5 d 6 s$ electrons. A subpicosecond access to the localized $4 f$ spin moments is therefore required, thus revealing the important role played by the $\mathrm{Gd}$ $4 f$ electrons in this fast reversal process. Such excitation time scale is indeed allowed in Gd by the strong exchange coupling between $5 d-4 f$, responsible for its ferromagnetic order $[28,29]$. The strength of this intra-atomic exchange is $\approx 100 \mathrm{meV}$, corresponding to $\approx 50 \mathrm{fs}$. From here, it follows that indeed the localized $4 f$ spin magnetic moment in Gd can be optically excited on a time scale comparable with that observed for the itinerant ferromagnets.
In summary, using a time-resolved magneto-optical technique, we have unambiguously demonstrated the feasibility of subpicosecond magnetization reversal in the ferrimagnetic alloy $\mathrm{GdFeCo}$. This ultrafast reversal time is a result of the divergence of the effective gyromagnetic ratio of the ferrimagnetic system at the angular momentum compensation temperature $T_{\mathrm{A}}$. These results also imply that the localized moment in Gd can be optically excited on a subpicosecond time scale. Besides its fundamental importance, the observation reported here might be of real interest for magnetic and magneto-optical recording employing rare-earth-transition-metal alloys [14].

The authors acknowledge the technical support of T. Toonen, W. Szweryn, and A. van Etteger and the financial support of The European network DYNAMICS, FOM, NWO, and the Grant in Aid for Scientific Research of the MEXT in Japan No. 16360182.

[1] E. Beaurepaire et al., Phys. Rev. Lett. 76, 4250 (1996).

[2] C. H. Back et al., Phys. Rev. Lett. 81, 3251 (1998).

[3] B. C. Choi et al., Phys. Rev. Lett. 86, 728 (2001).

[4] Th. Gerrits et al., Nature (London) 418, 509 (2002).

[5] H.W. Schumacher et al., Phys. Rev. Lett. 90, 017201 (2003).

[6] R. Hertel et al., Phys. Rev. Lett. 98, 117201 (2007).

[7] C. H. Back and D. Pescia et al., Nature (London) 428, 808 (2004).

[8] A. V. Kimel et al., Nature (London) 429, 850 (2004).

[9] K. Yu. Guslienko et al., Phys. Rev. B 70, 104405 (2004).

[10] G. Ju et al., Phys. Rev. Lett. 93, 197403 (2004).

[11] C. D. Stanciu et al., Phys. Rev. B 73, 220402(R) (2006).

[12] C. D. Stanciu et al., Phys. Rev. Lett. 98, 207401 (2007).

[13] D. Cimpoesu, A. Stancu, and L. Spinu, J. Appl. Phys. 102, 013915 (2007).

[14] Xin Jiang et al., Phys. Rev. Lett. 97, 217202 (2006).

[15] V. V. Randoshkin et al., Phys. Solid State 45, 513 (2003).

[16] Robert S. Weng and Mark H. Kryder, IEEE Trans. Magn. 29, 2177 (1993).

[17] R. K. Wangsness, Phys. Rev. 91, 1085 (1953).

[18] M. Aeschlimann et al., Appl. Phys. Lett. 59, 2189 (1991).

[19] M. Aeschlimann et al., J. Appl. Phys. 67, 4438 (1990).

[20] P. Chaudhari, J. J. Cuomo, and R. J. Gambino, Appl. Phys. Lett. 22, 337 (1973).

[21] A. F. Bartelt et al., Appl. Phys. Lett. 90, 162503 (2007).

[22] O. S. Anilturk and A. R. Koymen, Phys. Rev. B 68, 024430 (2003).

[23] M. Vomir et al., Phys. Rev. Lett. 94, 237601 (2005).

[24] J. Hohlfeld et al., Phys. Rev. B 65, 012413 (2001).

[25] H.-S. Rhie, H. A. Dürr, and W. Eberhardt, Phys. Rev. Lett. 90, 247201 (2003).

[26] A. Vaterlaus, T. Beutler, and F. Meier, Phys. Rev. Lett. 67, 3314 (1991).

[27] M. Binder et al., Phys. Rev. B 74, 134404 (2006).

[28] U. Bovensiepen, Appl. Phys. A 82, 395 (2006).

[29] I. Radu, Ph.D. thesis, Berlin, Germany, 2006. 